\title{
Investigating Direct Non-Age Metrics of Stored Blood Quality Loss
}

\author{
K Alfano, M Tarasev
}

\begin{abstract}
Citation
K Alfano, M Tarasev. Investigating Direct Non-Age Metrics of Stored Blood Quality Loss. The Internet Journal of Medical Technology. 2011 Volume 5 Number 1.
\end{abstract}

DOI: $\underline{10.5580 / 2789}$

\begin{abstract}
Long storage times for blood products are often unavoidable. Product age is essentially the only indicator used today for Red Blood Cell (RBC) quality loss during storage. Much controversy persists over the impact of RBC age on transfusion outcomes, as studies on this remain inconclusive. Such inconsistency may arise from unit-to-unit variability, which likely introduces some age-independence to RBC state. Thus, quality metrics other than storage time could aid with inventory management and/or treatment decisions. RBC membrane mechanical fragility is proposed here as one such candidate in vitro metric: it aggregately reflects a range of biochemical and biomechanical changes associated with storage lesion, and can provide a more comprehensive characterization of particular units than other properties. Preliminary data suggest this property can vary substantially among units of equal age, and further work now in progress is investigating its correlation to post-transfusion red cell survival in vivo.
\end{abstract}

\section{INTRODUCTION}

Transfusing Red Blood Cells (RBC) has been a common treatment for anemia since the introduction of preservative solutions in the mid- $20^{\text {th }}$ century. In severe hemorrhage cases, massive transfusions of up to 10-15 units are often required. For example, during 5 years of US military operations, about 86,000 units of RBC and whole blood (WB) were administered to about 5,000 patients $(1,2)$. While the benefit of transfusion for severe hemorrhage cases is well accepted, multiple studies suggest that RBC transfusions nevertheless can fall short of achieving their desired clinical effect - particularly for certain vulnerable patient groups - prompting reexamination of transfusion protocols and the preserved efficacy of stored packed RBC (see (3-8) for reviews).

Two main reasons have been proposed to account for reports of suboptimal transfusion efficacy: 1) adverse effects from non-RBC cells in transfused blood, and 2) changes in RBC resulting from prolonged storage - so-called "storage lesion" (9). Regarding the first, the presence of plasma factors and blood formed elements (e.g. leukocytes and platelets) was identified as potentially affecting in vivo performance of stored packed RBC $(6,10-13)$. Much evidence now demonstrates the benefits of leukocyte filtration (leukoreduction) for certain patient groups, although some controversy over this treatment still continues despite strong endorsement by the US Food and Drug Administration ((14-16)). In this article we discuss the second reason, RBC storage lesion, suggesting that age-independent metrics could enable better informed treatment and/or inventoryplanning decisions - particularly in situations where "fresh" blood is rarely even an option.

\section{BACKGROUND: STORAGE LESION AND THE AGE DEBATE}

Current civilian storage needs in the US result in an average RBC unit age of 21 days at transfusion (17), with the maximum permitted shelf life being 42 days. A survey of 1,500 American blood banks found that $20 \%$ of RBC units were $\geq 28$ days old $(18,19)$. For rare blood types such as ONegative, more than $60 \%$ of stored blood units were $\geq 28$ days old (20). On the military side, collected blood is processed into components and shipped by air to operation theaters; the increased complexity of providing regular RBC shipments to combat support hospitals results in units being aged 27-32 days upon delivery. By the time of transfusion, these units are 32 days and older, as reported by Spinella et.al. (21). 
RBC storage lesion encompasses various cellular changes associated with a host of metabolic and morphological changes in blood. In retrospective and prospective studies, these changes were linked with RBC damage, reduced posttransfusion survival/viability, and diminished ability of cells to deliver oxygen to tissues (see $(3,22,23)$ for reviews).

Morphological changes in RBC likely result from profound changes in the RBC membrane, including damage and redistribution of cytoskeletal proteins and membrane lipids (possibly correlated to unmitigated oxidative stress), membrane phospholipid vessiculation and loss, changes in membrane-bound carbohydrates, and loss of sialic acid (related to changes in electro-chemical RBC properties). These storage-induced processes translate into macro-scale effects including changes in shape, volume, and density along with greater membrane rigidity and cell aggregability (which may contribute to changes in blood viscosity and increased RBC-endothelial interactions). These may be further compounded by the increased levels of endotoxins and inflammatory cytokines associated with prolonged blood storage. An accumulation of pro-inflammatory bioactive compounds, a concordant decline in antioxidant capacity (altering the balance between pro-oxidant and anti-oxidant species), changes in glucose and potassium levels, and a rise in hemoglobin oxidation have also been observed. Decreases in ATP levels (typically a 20-25\% decline by the end of storage) have been documented as well, along with declines in 2,3-diphosphoglycerate (2,3-DPG) levels (essentially disappearing after 2 weeks of storage). It should be noted that despite their decline during storage, both ATP and 2,3DPG were shown to recover in vivo upon transfusion; however, this may not occur fast enough for critical patients in need of immediate restoration of oxygen delivery. (See $(22,24,25)$ for comprehensive reviews on storage lesion.)

Some investigators have reported that prolonged storage times were correlated with adverse clinical outcomes such as mortality, lengthened hospital stays, pneumonia, multiple organ failure, hemorrheological disorders, infections and sepsis, renal failure, TRALI, inflammatory responses, and microcirculatory and hemodynamic complications. On the other hand, other investigators did not find RBC age to adversely affect transfusion efficacy. The inconsistencies among findings render the issue yet unresolved, although various explanations have been proposed to reconcile the conflicts (see $(3,4,22-24,26)$ for reviews). Similarly, attempts to quantify certain in vitro properties associated with storage lesion have yielded wide inconsistencies as well, although this was likely due to unvalidated analytical methods and a lack of common standards (27). And notably, most prospective studies comparing efficacies of differentlyaged units look only at blood within the first half of its shelf life, leaving the latter half largely unexplored.

In 2010 alone, the issue of storage lesion and its potential impact on transfusion outcomes has been the subject of over a dozen literature reviews (including $(23,28-30)$ ). The ageof-blood debate has also attracted public attention, with a recent Wall Street Journal article highlighting the controversy (31). However, age is only one contributing factor to blood quality - hence the potential usefulness of non-age metrics.

\section{AGE ALONE AN INADEQUATE PROXY FOR QUALITY}

The widespread use of First-In-First-Out (FIFO) inventory methods, and reliance upon a universal shelf life, makes storage time or age the exclusive de facto metric of storage lesion today. However, it has been shown that the magnitudes of observed changes can significantly depend upon the unit manufacturer, as well as transport and storage issues (including type of A-P solution used, presence of modifying additives, and position of the bag during storage) (32-35). The unit-to-unit variability may also be further compounded by differing amounts of leucocytes and platelets in the solution (10).

Differences among RBC coming from different donors is another very important consideration (36). Generally the notion of RBC "age" only considers time since collection, but the mean red cell age at donation can span from 38 to 60 days (37). Notably, inter-donor variability was shown to be the most significant contributing factor for in-bag hemolysis ("autolysis") of stored packed RBC (38). The link between RBC age within donors and storage lesion was further corroborated recently by Sparrow et al. $(39,40)$, whose results indicated that $\mathrm{RBC}$ age upon donation can influence the nature and the magnitude of storage lesion - possibly by affecting the carbohydrate structures of the cell membrane (40). Potential dependence of in-bag hemolysis on donorspecific factors has been observed by McAteer (41), and Buchwald recently demonstrated that oxygen transport rates are consistent for each whole-blood donor while varying across different donors (42). Correlation between storage time and blood quality is further eroded by reported dependency upon donor gender and age, and storage solution used (43). 
Considering this, it is not surprising that studies investigating the impact of RBC age on transfusion outcomes are inconsistent. It thus becomes worthwhile to broaden the scope of inquiry beyond storage time, including investigation of possible in vitro tools for evaluating stored packed RBC.

\section{RESEARCHING CANDIDATE METRIC(S)}

It remains to be proven which if any in vitro measures, individually or in combination, can be reliably correlated with transfusion efficacy. Certain cell membrane properties - many loosely termed "deformability," despite significant variations - show potential to reflect aggregations of phenomena associated with storage lesion. For example, changes in ATP levels have been correlated to membrane changes, as some RBC membrane functions are energydependent and thus partly dependent upon metabolic state. While the active preservation of cell ATP levels did slow some indicia of storage lesion, the correlation between cell ATP and transfusion outcomes has been shown only for extremely depleted cells. Nevertheless, whatever the role ATP depletion does play in cell performance can potentially be captured via membrane properties. More directly, a loss of endogenous RBC antioxidants results in oxidative damage to cytoskeletal proteins and membrane phospholipids causing measurable changes in membrane properties (see (4, 22) for reviews). Moreover, many other biomechanical effects of storage lesion are either causes or effects of membrane changes.

On the clinical side, altered membrane properties have been correlated with diminished oxygen transport, and arguably with post-transfusion complications. RBC membrane rigidity can impede the cells' ability to navigate the microcirculatory network - hindering restoration of tissue oxygenation. Microvascular flow can be further compromised by occlusion of microcirculation by rigid cells and/or by membrane-mediated RBC-endothelial interactions. Altered mechanical properties of the RBC membrane are also likely responsible for the cell lysis shown to occur inbag during storage and suggested to occur in vivo posttransfusion. In addition, RBC age and associated membrane changes were implicated in high post-transfusion free $\mathrm{Hb}$ levels in trauma patients (44). Such release of free $\mathrm{Hb}$ into the bloodstream can overload the capacity of haptoglobin (responsible for free $\mathrm{Hb}$ removal), resulting in hemoglobinemia (45), redox injury of endothelium or underlying tissues, or depletion of nitric oxide (46). Furthermore, it has been suggested that limited nitric oxide bioavailability causes regional and systemic vasoconstriction and subsequent organ dysfunction $(47,48)$. Alterations to RBC membranes are also thought to trigger immune system mechanisms for RBC removal - increasing the clearance of transfused cells from circulation - which would be of greatest concern for chronic anemia patients (see $(4,9,22$, 25,49 ) for reviews).

To now distinguish among particular RBC membrane properties, we specifically single out mechanical fragility in contrast to either "deformability" or osmotic fragility. While fragility testing involves stressing a sample of cells until some fraction thereof lyse, deformability testing typically measures a sample's (average) ability to be manipulated without lysing. Deformability testing has long been pursued in clinical diagnostics, and fragility testing occurs in research settings, but as yet no such metrics have been implemented for systematic evaluation of RBC storage lesion or degradation.

Regarding mechanical fragility, one limitation on its adoptability for standardized clinical applications appears to have been a lack of a testing means that is both highly sensitive and clinically practical (50). However, we believe this erythrocyte property to be a particularly likely measure of membrane state to correlate with transfusion efficacy: Mechanical fragility represents a sample's propensity for hemolysis under sustained and varied physical stresses offering greater potential, with proper configuration, to resemble the physiological stresses cells experience in vivo. Secondly, the nature of mechanical fragility testing offers the novel advantage of being able to vary multiple stress parameters concurrently for complexly sensitive multidimensional fragility profiles (discussed below). Thirdly, unlike typical "deformability" measurements, profiling a sample's mechanical fragility allows the entire distribution of a unit's population and sub-populations to be represented, for a more comprehensive evaluation of each individual unit.

Notably, a recent study showed that mechanical fragility is correlated, independently of age, with factors such as storage solution and donor gender (43).

\section{OUR SYSTEM AND PRELIMINARY DATA}

Our proprietary system (51) involves three integrated steps being performed on a sample of stored blood product: 1) subjection of the sample to a range of controlled physical stresses using multiple parameters (e.g., intensity, duration), 2) spectrophotometric determination of the extent of hemolysis occurring under various combinations of stress 
parameters, and 3) computational processing of the results (e.g., lysis levels and rates) to comprehensively quantify the sample via a multi-dimensional fragility profile matrix. And for the test to be performed more rapidly and easily, the spectrophotometric step can be achieved using a proprietary optical unit uniquely capable of collecting the relevant spectra in real time without requiring centrifugation or intermediary handling (52).

\section{Figure 1}
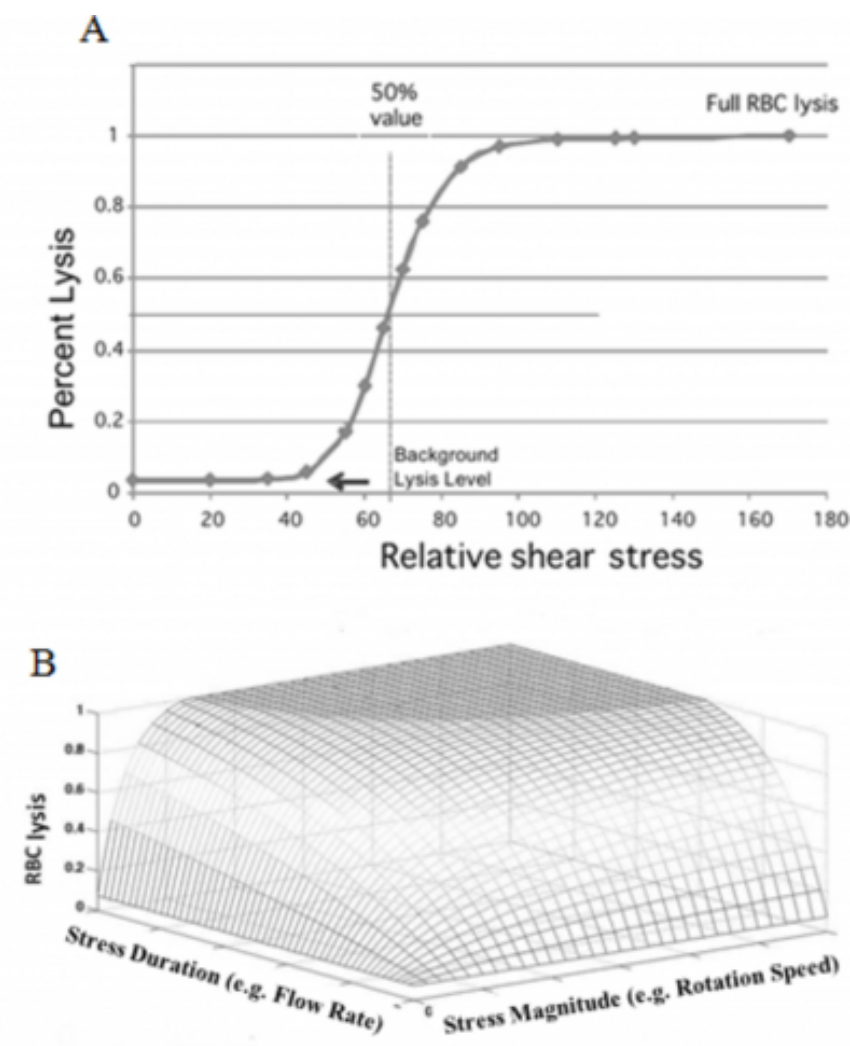

Figure 1: Conceptual 2D (A) and 3D (B) fragility profile models for RBC. A 3D depiction enables graphing lysis dependence upon two selected stress parameters, e.g. intensity and duration. Background lysis is the in-bag "autolysis" of RBC, represented at zero applied extemal stress. (A small sampling of RBC is tested for auto-lysis upon outdating - regulated with a $1 \%$ maximum in the US.)

With a single parameter of total stress magnitude, fragility is conventionally profiled as a 2D sigmoidal function (as shown in Figure 1A). However, our approach separates out parameters such as stress intensity and stress duration to create a 3D fragility profile (as shown in Figure 1B). Such parameters independently contribute to total shear stress, so separating them is expected to enrich fragility characterizations. Several other stress parameters (e.g., iterations, qualitative stress types/components, etc.) can be added and separated for increasingly sophisticated profiles via higher-dimensional matrices.

\section{Figure 2}

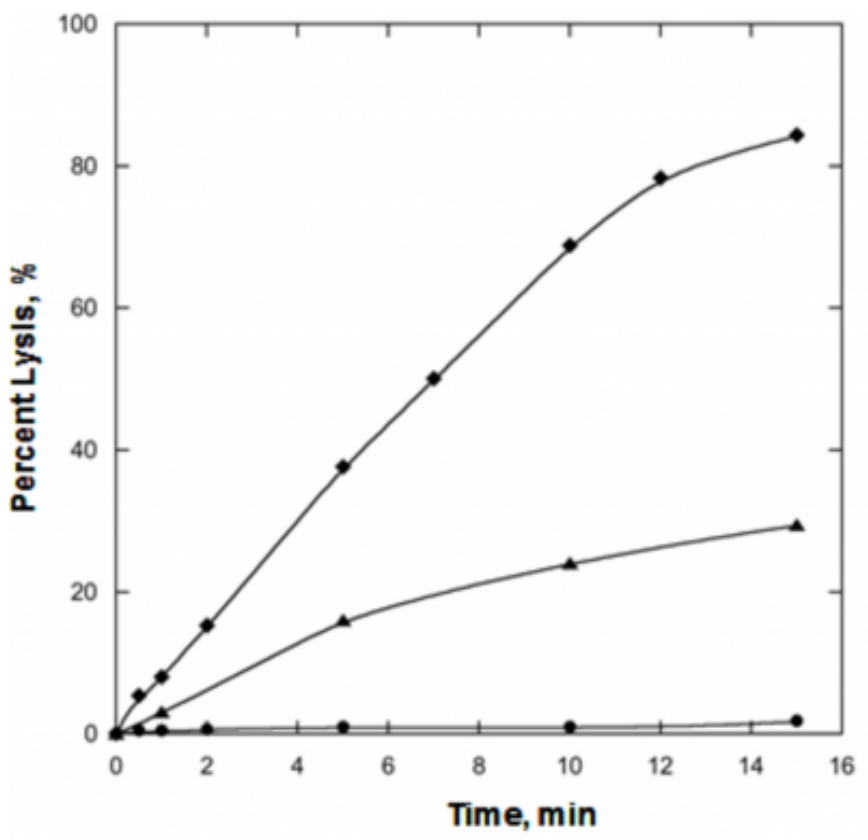

Figure 2: Dependence of induced RBC lysis upon shear stress duration and intensity. Undiluted RBC samples of $1.5 \mathrm{ml}$ obtained from one unit ( 21 days before expiration) were each lysed using a TissueLyser LT (Quiagen) with a single $5 \mathrm{~mm}$ steel bead. Samples were lysed at oscillation frequencies of $50(\boldsymbol{\bullet}), 35(\boldsymbol{\Delta})$ and $15(\boldsymbol{\bullet}) \mathrm{Hz}$. After brief centrifugation, aliquots of supernatant were diluted as needed with $0.1 \mathrm{M}$ HEPES, $\mathrm{pH} 7.8$, and the amounts of released hemoglobin $(\mathrm{Hb})$ were measured using a NanoDrop ND100 spectrophotometer based on the $A_{577}-$ A 700 absorbance difference. Results are presented as fractions (percent lysis) of $\mathrm{Hb}$ released into the supernatant compared to known total $\mathrm{Hb}$ content as determined via repeated freeze-thaw of a sample using liquid nitrogen.

Various complex changes can occur over time to a fragility profile. Such changes can include an increase in the mean cell fragility of a sample, an increase in the standard deviation (for a normally-distributed population), deviations from normality, and/or the development of cell subpopulations with distinct profiles. This wide range of potential changes allows highly-sensitive and complex characterizations - in turn enabling the study of many possible clinical correlations.

While integrated custom instrumentation remains under development, we are initially testing the concepts using a commercially available bead mill (TissueLyser LT from Quiagen) and a NanoDrop ND100 spectrophotometer. The TissueLyser subjects a sample to shear stress with the 
variable parameters of oscillation frequency and duration.

Figure 2 illustrates the dependence of RBC lysis levels upon applied stress intensity (Hz) and duration (min). As

anticipated, higher stress resulted in greater lysis as reflected in the resultant concentrations of free hemoglobin. Arrays of 2D profiles, similar to those in Figure 2, could be aggregated to construct $3 \mathrm{D}$ profiles.

\section{Figure 3}

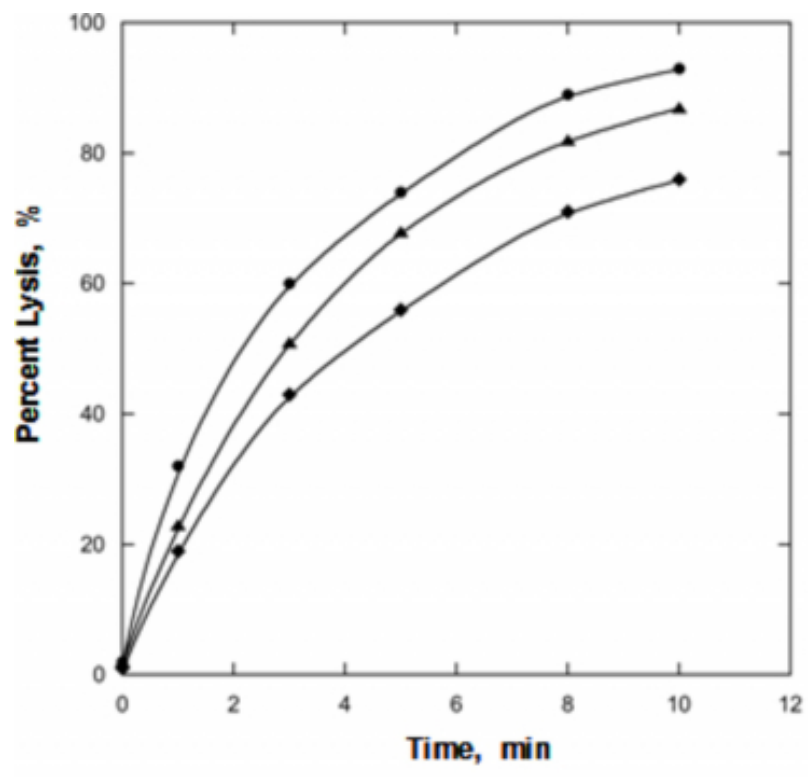

Figure 3: Example of variability in fragility among RBC units of the same age ( 3 shown of 6 tested). RBC samples obtained from unit test strips (10 days before expiration) were diluted prior to lysis to $25 \mu \mathrm{M}$ total hemoglobin concentration with AS3 storage solution supplemented with $30 \mathrm{~g} / \mathrm{L}$ bovine serum albumin. Lysed samples were $350 \mathrm{uL}$, and no post-lysis dilution was performed. Otherwise, lysis and subsequent spectral analysis were conducted similarly to as for Figure 2. Each data point represents 3 independent measurements; average relative standard error $=4 \%$, excluding measurements at "zero" duration (in-bag lysis alone) for which average relative standard error $=$ $10 \%$.

Preliminary bench-top data obtained from RBC units of the same age and before expiration are presented in Figure 3 (3 units shown of 6 tested). The lysis step used varying stress durations with a fixed intensity of $50 \mathrm{~Hz}$. Results indicate that among 6 randomly-selected RBC units of the same age, there was statistically significant variability in mechanical fragility which was reproducibly detected. One simple way to describe such variability is by $S_{50}$, the amount of stress necessary to lyse $50 \%$ of the cells in a sample $\mathrm{K}_{50}$ is the slope at this point. For all 6 units tested, quadratically- interpolated $\mathrm{S}_{50}$ and corresponding $\mathrm{K}_{50}$ values are listed in Table 1; the data also suggest some potential independence between these two values. While such characteristics permit straightforward comparisons, more complex profiles may reveal more meaningful patterns in stress susceptibilities.

\section{Figure 4}

\section{Table 1. $\mathrm{S}_{50}$ and $\mathrm{K}_{50}$ values for the 6 units tested. Data is \pm 0.1 .}

\begin{tabular}{|l|l|}
\hline $\mathrm{S}_{50, \min }$ & $\mathrm{K}_{50,} \% / \mathrm{min}$ \\
\hline 4.0 & 8.5 \\
\hline 3.8 & 9.2 \\
\hline 3.0 & 11.5 \\
\hline 3.0 & 11.0 \\
\hline 2.0 & 14.5 \\
\hline 2.5 & 13.6 \\
\hline
\end{tabular}

\section{POTENTIAL APPLICATIONS UPON VALIDATION}

Individualized unit data regarding levels and rates of RBC lesion, representing relative preservation of in vivo efficacy, could enable a quality-based approach to inventory management (analogous to Shortest-Remaining-Shelf-Life (SRSL) for perishable foods (53)) to supplement or replace FIFO. The breadth and frequency of testing in an inventory would depend on the clinical value of information gained for the cost. Upon gathering unit-specific data, supply and inventory management principles could be employed to improve utilization: For example, a unit whose prospective efficacy is declining more rapidly than others may get used sooner, to minimize quality loss. This could improve transfusion efficacy overall, and thus lower rates of post- 
transfusion complications - and adverse reaction costs (54). This could have the net effect of conserving blood, particularly when there is a wider range of unit ages to work with. And when such inventory management flexibility is not feasible, testing a unit at the point of collection may provide useful information on its likely longevity before its destination is decided.

Also, a triaging application could potentially enable clinicians to prioritize the highest-performing units available for those patients presenting greatest need. Testing could occur regularly across an inventory, or on-demand as deemed warranted. Presently, such prioritizing of units perceived to have greatest efficacy is done only for neonatal patients, and this is based solely on storage time ( $<7$ days).

Alternatively, it is conceivable that some units which may be ineffective for some patients could nevertheless be beneficial for others; for example, as suggested by Vincent et al. (55), some RBC units which may be ineffective for patients having normal erythrocyte deformability could nevertheless be beneficial for patients having altered deformability (particularly when correcting a small oxygen deficit). The multi-dimensional profiling approach may ultimately reveal that "quality" is a more complex notion than a single continuum - meaning the most desirable RBC profile may in fact depend upon particular patient needs.

\section{CLINICAL VALIDATION AND FUTURE WORK}

We are presently beginning our first clinical study correlating RBC mechanical fragility in vitro to posttransfusion cell survival in vivo. Success in this study will enable larger-scale follow-up studies, with expansion to include additional clinical outcomes beyond cell survival (such as tissue oxygenation). At each step, defining performance metrics and selecting appropriate patient groups will require continued and additional medical collaborations. Despite the complexity and scope of the undertaking, there is precedent for such studies eventually achieving broad acceptance (e.g. TRICC Trial (56)).

Initial versions of the marketed device will simply provide new information to blood banking and transfusion physicians regarding existing inventories, so US commercialization under a general usage indication is anticipated to be as an FDA Class 2 device via either a $510(\mathrm{k})$ or $513(\mathrm{f})$ pathway. The low-risk device involves no patient contact or product alteration, and does not require using any units that are not already approved (or for any patient who could not already receive them today).
Additional studies may later seek to justify specific usage claims regarding inventory protocols and/or clinical triage.

\section{CONCLUSION}

While the need to store $\mathrm{RBC}$ remains necessary for the foreseeable future, steps can and should be taken to most effectively utilize the limited supply. Additional measures of blood quality beyond age alone are needed to more accurately characterize stored blood product, particularly when long storage times are unavoidable. A clinicallyfeasible test of RBC membrane mechanical fragility as a supplemental quality metric could aid medical professionals in making more informed decisions.

\section{ACKNOWLEDGMENTS}

The authors appreciate the support of Robertson Davenport, MD, Medical Director of the University of Michigan Hospital Blood Bank, who has provided valuable advice as well as RBC units and segments for experimentation.

\section{PERSONAL INTEREST DISCLOSURE}

The authors are employed with Blaze Medical Devices - an Ann Arbor, Michigan-based start-up company pursuing commercialization of the proprietary technology introduced in this article.

\section{References}

1. Hess JR, Holcomb JB: Transfusion practice in military trauma. Transfus Med; 2008; 18: 143-50.

2. Borgman MA, Spinella PC, Perkins JG, Grathwohl KW, Repine T, Beekley AC, et al.: The ratio of blood products transfused affects mortality in patients receiving massive transfusions at a combat support hospital. J Trauma; 2007; 63: 805-13.

3. Tinmouth A, Chin-Yee I: The clinical consequences of the red cell storage lesion. Transfus Med Rev; 2001; 15: 91-107. 4. Ho J, Sibbald WJ, Chin-Yee IH: Effects of storage on efficacy of red cell transfusion: when is it not safe? Crit Care Med; 2003; 31: S687-97.

5. Napolitano LM, Corwin HL: Efficacy of red blood cell transfusion in the critically ill. Crit Care Clin; 2004; 20:

255-68.

6. Madjdpour C, Spahn DR: Allogeneic red blood cell transfusions: efficacy, risks, alternatives and indications. $\mathrm{Br}$ J Anaesth; 2005; 95: 33-42.

7. Marik PE, Corwin HL: Efficacy of red blood cell transfusion in the critically ill: a systematic review of the literature. Crit Care Med; 2008; 36: 2667-74.

8. Napolitano LM, Kurek S, Luchette FA, Corwin HL, Barie PS, Tisherman SA, et al.: Clinical practice guideline: red blood cell transfusion in adult trauma and critical care.

Critical Care Medicine; 2009; 37: 3124-57.

9. Hebert PC, Tinmouth A, Corwin HL: Controversies in RBC transfusion in the critically ill. Chest; 2007; 131:

1583-90.

10. Greenwalt TJ, Zehner Sostok C, Dumaswala UJ: Studies in red blood cell preservation. 1. Effect of the other formed elements. Vox Sang; 1990; 58: 85-9. 
11. Wortham ST, Ortolano GA, Wenz B: A brief history of blood filtration: clot screens, microaggregate removal, and leukocyte reduction. Transfus Med Rev; 2003; 17: 216-22. 12. Roddie PH, Turner ML, Williamson LM: Leucocyte depletion of blood components. Blood Rev; 2000; 14: 145-56.

13. Seghatchian MJ: An overview of laboratory and clinical aspects of leucocyte-depleted blood components. Transfus Sci; 1994; 15: 49-56.

14. Tasaki T, Ohto H, Sasaki S, Kanno T, Igari T, Hoshi Y: Significance of pre-storage leucoreduction for autologous blood. Vox Sang; 2009; 96: 226-33.

15. Seghatchian J: Universal leucodepletion: an overview of some unresolved issues and the highlights of lessons learned. Transfus Apher Sci; 2003; 29: 105-17.

16. Dzik WH: Leukoreduction of blood components. Curr Opin Hematol; 2002; 9: 521-6.

17. Corwin HL, Gettinger A, Levy MM, Fink MP, Pearl RG, Abraham Eea: Anemia and blood transfusion in the critically ill: curent clinical practice in the United States - The CRIT

Study. Crit Care Med; 2004; 32: 39-52.

18. Vamvakas EC, Carven JH: Length of storage of transfused red cells and postoperative morbidity in patients undergoing coronary artery bypass graft surgery.

Transfusion; 2000; 40: 101-9.

19. Goldman M, Blajchman MA. Bacterial contamination. In: Popovsky MA, editor. Transfusion reactions. Bethesda: Americal Association of Blood Banks Press; 1996. p. 125-65.

20. Owens W, Tokessey M, Rock G: Age of blood in inventory at a large tertiary care hospital. Vox Sang; 2001; 81: 21-3.

21. Spinella PC, Perkins JG, Grathwohl KW, Repine T, Beekley AC, Sebesta J, et al.: Risks associated with fresh whole blood and red blood cell transfusions in a combat support hospital. Crit Care Med; 2007; 35: 2576-81. 22. Almac E, Ince C: The impact of storage on red cell function in blood transfusion. Best Pract Res Clin Anaesthesiol; 2007; 21: 195-208.

23. Zubair AC: Clinical impact of blood storage lesions. Am J Hematol; 2010; 85: 117-22.

24. Tinmouth A, Fergusson D, Yee IC, Hebert PC: Clinical consequences of red cell storage in the critically ill.

Transfusion; 2006; 46: 2014-27.

25. Frenzel T, Westphal-Varghese B, Westphal M: Role of storage time of red blood cells on microcirculation and tissue oxygenation in critically ill patients. Curr Opin

Anaesthesiol; 2009; 22: 275-80.

26. Napolitano LM, Corwin HL: Efficacy of blood

transfusion in the critically ill: does age of blood make a

difference? Crit Care Med; 2004; 32: 594-5.

27. Hess JR, Kagen LR, van der Meer PF, Simon T,

Cardigan R, Greenwalt TJ, et al.: Interlaboratory comparison of red-cell ATP, 2,3-diphosphoglycerate and haemolysis measurements. Vox Sang; 2005; 89: 44-8.

28. Glynn SA: The red blood cell storage lesion: a method to the madness. Transfusion; 2010; 50: 1164-9.

29. Hess JR: Red cell storage. Journal of Proteomics; 2010; 73: 368-73.

30. Triulzi DJ, Yazer MH: Clinical studies of the effect of blood storage on patient outcomes. Transfusion \& Apheresis Science; 2010; 43: 95-106.

31. Wang SS. What's the Shelf Life of Blood? Focus on Whether Older Donations Impair Recovery of Transfusion Recipients. The Wall Street Journal. 2009 Dec. 1.

32. Gyongyossy-Issa MI, Weiss SL, Sowemimo-Coker SO, Garcez RB, Devine DV: Prestorage leukoreduction and lowtemperature filtration reduce hemolysis of stored red cell concentrates. Transfusion; 2005; 45: 90-6.

33. Hogman CF, Eriksson L, Gong J, Hogman AB, Payrat JM: Shall red cell units stand upright, lie flat or be mixed during storage? In vitro studies of red cells collected in 0.5 CPD and stored in RAS2 (Erythrosol). Transfus Sci; 1995; 16: 193-9.

34. Holme S: Current issues related to the quality of stored RBCs. Transfus Apher Sci; 2005; 33: 55-61.

35. Zimmermann R, Heidenreich D, Weisbach V, Zingsem J, Neidhardt B, Eckstein R: In vitro quality control of red blood cell concentrates outdated in clinical practice.

Transfus Clin Biol; 2003; 10: 275-83.

36. Card RT, Mohandas N, Mollison PL: Relationship of post-transfusion viability to deformability of stored red cells. Br J Haematol; 1983; 53: 237-40.

37. Cohen RM, Franco RS, Khera PK, Smith EP, Lindsell CJ, Ciraolo PJ, et al.: Red cell life span heterogeneity in hematologically normal people is sufficient to alter $\mathrm{HbA} 1 \mathrm{c}$. Blood; 2008; 112: 4284-91.

38. Hess JR, Sparrow RL, van der Meer PF, Acker JP, Cardigan RA, Devine DV: Red blood cell hemolysis during blood bank storage: using national quality management data to answer basic scientific questions. Transfusion; 2009; 49: 2599-603.

39. Sparrow RL, Healey G, Patton KA, Veale MF: Red blood cell age determines the impact of storage and leukocyte burden on cell adhesion molecules, glycophorin A and the release of annexin V. Transfus Apher Sci; 2006; 34: $15-23$.

40. Sparrow RL, Veale MF, Healey G, Payne KA: Red blood cell (RBC) age at collection and storage influences RBC membrane-associated carbohydrates and lectin binding. Transfusion; 2007; 47: 966-8.

41. McAteer MJ, Dumont LJ, Cancelas J, Rugg N, Vassallo $\mathrm{R}$, Whitley $\mathrm{P}$, et al.: Multi-institutional randomized control study of haemolysis in stored red cell units prepared manually or by an automated system. Vox Sanguinis; 2010; 99: 34-43.

42. Buchwald H, Menchaca HJ, Michalek VN, Rudser KD, Rohde TD, O'Dea T, et al.: Pilot study of oxygen transport rate of banked red blood cells. Vox Sang; 2009; 96: 44-8. 43. Raval JS, Waters JH, Seltsam A, Scharberg EA, Richter E, Daly AR, et al.: The use of the mechanical fragility test in evaluating sublethal RBC injury during storage. Vox Sang; 2010; 99: 325-31.

44. Nishiyama T, Hanaoka K: Free hemoglobin concentrations in patients receiving massive blood transfusion during emergency surgery for trauma. Can J Anaesth; 2000; 47: 881-5.

45. Sowemimo-Coker SO: Red blood cell hemolysis during processing. Transfus Med Rev; 2002; 16: 46-60.

46. Napolitano L: Cumulative risks of early red blood cell transfusion. J Trauma; 2006; 60: S26-34.

47. Gladwin MT, Crawford JH, Patel RP: The biochemistry of nitric oxide, nitrite, and hemoglobin: role in blood flow regulation. Free Radic Biol Med; 2004; 36: 707-17. 48. Reiter CD, Gladwin MT: An emerging role for nitric oxide in sickle cell disease vascular homeostasis and therapy. Curr Opin Hematol; 2003; 10: 99-107. 49. Scharte M, Fink MP: Red blood cell physiology in critical illness. Crit Care Med; 2003; 31: S651-7. 50. Gu L, Smith WA, Chatzimavroudis GP: Mechanical fragility calibration of red blood cells. Asaio J; 2005; 51: 194-201.

51. Alfano K, Tarasev M (2009) An Apparatus and Methods to Characterize Blood and Red Blood Cells via Erythrocyte Membrane Fragility Quantification, US Patent Application Number 12690916. 
52. Tarasev M (2007) Blood Hemolysis Analyzer, US Patent Number 7790464.

53. Wells JH, Singh RP: A quality-based inventory issue policy for perishable foods. Journal of Food Processing and Preservation; $1989 ; 12:$ 271-92.

54. Shander A, Hofmann A, Gombotz H, Theusinger OM, Spahn DR: Estimating the cost of blood: past, present, and future directions. Best Pract Res Clin Anaesthesiol; 2007; 21: 271-89.
55. Vincent JL, Sakr Y, De Backer D, Van der Linden P: Efficacy of allogeneic red blood cell transfusions. Best Pract Res Clin Anaesthesiol; 2007; 21: 209-19.

56. Hebert PC, Wells G, Blajchman MA, Marshall J, Martin C, Pagliarello G, et al.: A multicenter, randomized, controlled clinical trial of transfusion requirements in critical care. Transfusion Requirements in Critical Care

Investigators, Canadian Critical Care Trials Group. N Engl J Med; 1999; 340: 409-17. 


\section{Author Information}

Kenneth M. Alfano, MS, JD

University of Michigan and Blaze Medical Devices

Michael Tarasev, PhD, MBA

University of Michigan and Blaze Medical Devices 